\title{
FOXA1 deletion in luminal epithelium causes prostatic hyperplasia and alteration of differentiated phenotype
}

\author{
David J DeGraff ${ }^{1,11}$, Magdalena M Grabowska ${ }^{2,11}$, Tom C Case ${ }^{2}$, Xiuping Yu², Mary K Herrick², William J Hayward², \\ Douglas W Strand ${ }^{2}$, Justin M Cates ${ }^{3}$, Simon W Hayward ${ }^{2}$, Nan Gao ${ }^{4}$, Michael A Walter ${ }^{5}$, Ralph Buttyan ${ }^{6}$, Yajun Yi $^{7}$, \\ Klaus H Kaestner ${ }^{8}$ and Robert J Matusik ${ }^{1,9,10}$
}

The forkhead box (Fox) superfamily of transcription factors has essential roles in organogenesis and tissue differentiation. Foxa1 and Foxa2 are expressed during prostate budding and ductal morphogenesis, whereas Foxa1 expression is retained in adult prostate epithelium. Previous characterization of prostatic tissue rescued from embryonic Foxa1 knockout mice revealed Foxa1 to be essential for ductal morphogenesis and epithelial maturation. However, it is unknown whether Foxa1 is required to maintain the differentiated status in adult prostate epithelium. Here, we employed the PBCre4 transgenic system and determined the impact of prostate-specific Foxa1 deletion in adult murine epithelium. $\mathrm{PBCre} 4 /$ Foxa $1^{\text {loxp/loxp }}$ mouse prostates showed progressive florid hyperplasia with extensive cribriform patterning, with the anterior prostate being most affected. Immunohistochemistry studies show mosaic Foxa1 KO consistent with PBCre4 activity, with Foxa1 KO epithelial cells specifically exhibiting altered cell morphology, increased proliferation, and elevated expression of basal cell markers. Castration studies showed that, while PBCre4/Foxa $1^{\text {loxp/loxp }}$ prostates did not exhibit altered sensitivity in response to hormone ablation compared with control prostates, the number of Foxa1-positive cells in mosaic Foxa1 KO prostates was significantly reduced compared with Foxa1-negative cells following castration. Unexpectedly, gene expression profile analyses revealed that Foxa1 deletion caused abnormal expression of seminal vesicle-associated genes in KO prostates. In summary, these results indicate Foxa1 expression is required for the maintenance of prostatic cellular differentiation.

Laboratory Investigation (2014) 94, 726-739; doi:10.1038/labinvest.2014.64; published online 19 May 2014

KEYWORDS: androgen; AR; castration; prostate; prostate cancer; prostate-specific; FOXA

The Forkhead Box (Fox in mice, FOX in human) family of transcription factors consists of 43 proteins, which are organized into 19 subfamilies based on amino-acid sequence conservation. ${ }^{1}$ Foxal (originally termed hepatocyte nuclear factor one alpha; HNF1 $\alpha$ ) was originally discovered in mammals as a transcription factor in the liver. ${ }^{2}$ Importantly, the structure of the DNA-binding domain of Foxa proteins contains a variant of the helix-loop-helix motif, giving it the appearance of a 'winged helix.' This structure closely resembles that of a linker histone ${ }^{3}$ and enables Foxal to directly influence chromatin structure. In addition, the $\mathrm{C}$ terminus of Foxal binds to core histones and is required for chromatin opening in vitro. ${ }^{4}$ For these reasons, Foxa proteins make DNA sequences accessible for the binding of additional transcriptional activators and/or repressors, and have accordingly been described as 'pioneer factors.' The 'pioneering' function of Foxa proteins has led to the suggestion that these proteins enable cells to respond quickly to changes in their environment by altering transcriptional regulation of genes. The ability of Foxa transcription factors to impart 'competence' for the rapid initiation of gene expression has been suggested as central to their function. In addition, the observation that Fox family members are retained on mitotic chromosomes, ${ }^{5,6}$ suggests an important role for Foxal in the maintenance of cell fate.

\footnotetext{
'Department of Pathology, Pennsylvania State University, Hershey, PA, USA; ${ }^{2}$ Urologic Surgery, Vanderbilt University Medical Center, Nashville, TN, USA; ${ }^{3}$ Department of Pathology, Vanderbilt University Medical Center, Nashville, TN, USA; ${ }^{4}$ Department of Biological Sciences, Rutgers University, Newark, NJ, USA; ${ }^{5}$ Department Medical Genetics, University of Alberta, Edmonton, AB, USA; ${ }^{6}$ Vancouver Prostate Centre, Vancouver, BC, USA; ${ }^{7}$ Institute for Integrative Genomics and Department of Medicine, Vanderbilt University, Nashville, TN, USA; ${ }^{8}$ Department of Genetics, University of Pennsylvania, Philadelphia, PA, USA; ${ }^{9}$ Department of Cell and Developmental Biology, Vanderbilt University, Nashville, TN, USA and ${ }^{10}$ Vanderbilt-Ingram Cancer Center, Vanderbilt University Medical Center, Nashville, TN, USA

Correspondence: RJ Matusik, PhD, Director of Urologic Surgery Research, William L. Bray Chair of Urologic Surgery, Vanderbilt University Medical Center, 116121 st Avenue South, Nashville, TN 37232, USA.

E-mail: robert.matusik@vanderbilt.edu

${ }^{11}$ These authors contributed equally to this work.

Received 14 December 2013; revised 20 February 2014; accepted 26 March 2014
} 
Foxa1 and Foxa2 expressions are restricted to the epithelial compartment during embryonic prostate development. ${ }^{7}$ Whereas Foxa2 expression is extinguished following budding morphogenesis, Foxal expression is maintained in the adult prostate epithelium. Our laboratory previously showed that Foxal expression is required for normal prostate organogenesis. ${ }^{8,9}$ In fact, the expression of Foxa proteins have an essential role during the development of several organs, and Foxa1 and Foxa2 have been shown to act in a cooperative manner during the normal development of the liver, lungs, dopaminergic neurons, and pancreas..$^{10}$ Our laboratory was the first to report the direct physical interaction of Foxal with the androgen receptor (AR) and the importance of Foxal expression for the regulation of prostate-specific gene expression. ${ }^{11}$ While altered levels of Foxa1 and Foxa2 expression have been implicated in prostate cancer, ${ }^{12-17}$ the importance of Foxal expression in the adult, non-neoplastic prostate is unknown. Therefore, we undertook this study to determine the impact of prostate-specific genetic ablation of Foxal in adult mice.

\section{MATERIALS AND METHODS}

\section{Mouse Lines and Breeding}

Foxal ${ }^{\text {loxp }}$ mice have been previously described. ${ }^{18}$ PBCre $4^{19}$ activity was detected 14 days after birth and increases with rising testicular androgen production and the onset of sexual maturity in mice. PBCre4 has been previously reported to result in prostate-specific Cre recombinase activity, and Gt(ROSA)26Sor ${ }^{\text {tm4(ACTB-tdTomato,-EGFP)Luo/J }}$ mice (Jackson Laboratories, Bar Harbor, MA) express red fluorescence before, and green fluorescence following Cre-mediated recombination. ${ }^{20}$ To identify specific cell populations targeted by Cre-mediated recombination in the PBCre4 mouse line, PBCre4 mice were bred with

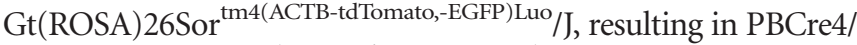

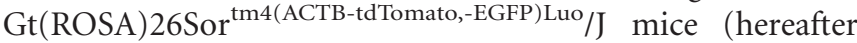
designated PBCre4/Tomato mice). Prostate-specific Foxal knockout was achieved by breeding PBCre4 mice to Foxal ${ }^{\text {loxp }}$ and Foxal $1^{\text {loxp/loxp }}$ mice, resulting in PBCre4/Foxal ${ }^{\text {loxp }}$ and PBCre4/Foxa1 $1^{\text {loxp/loxp }}$ mice, respectively. PBCre4/Foxal ${ }^{\text {loxp }}$ and PBCre4/Foxal ${ }^{\text {loxp/loxp }}$ mice were aged up to 40 weeks. Following sacrifice, individual prostate lobes, seminal vesicle, and bladders were dissected, formalin fixed, and processed for paraffin embedding according to standard procedures.

\section{Immunohistochemistry and Immunofluorescence}

Slides were deparaffinized, rehydrated through a series of graded alcohols, and washed in double deionized water for $5 \mathrm{~min}$. Tissues were then placed in antigen unmasking solution (Vector Labs, Burlingame, CA), and antigen retrieval was performed by microwaving samples for $20 \mathrm{~min}$ at $30 \%$ power in a $900 \mathrm{~W}$ microwave oven. Slides were then cooled to room temperature, and then washed three times for $10 \mathrm{~min}$ in PBS (pH 7.4). For immunohistochemistry, all incubations were performed at room temperature unless otherwise stated. Endogenous peroxidase activity was blocked with the use of Peroxidase blocking reagent (Dako North America, Carpinteria, CA) for $20 \mathrm{~min}$, after which sections were again washed in PBS three times for $10 \mathrm{~min}$. Prepared slides were incubated in horse serum for $30 \mathrm{~min}$ to reduce non-specific antibody binding. Slides where then incubated overnight at $4{ }^{\circ} \mathrm{C}$ in a humidified chamber with the primary antibody. After three 10-min washes in PBS, slides were then incubated overnight in the presence of primary antibody. On the following day, slides were then washed three times for 10 min with PBS and biotinylated secondary antibody diluted in PBS was then added. Primary antibody was visualized using the Vectastain Elite ABC Peroxidase kit (Vector Labs) according to the manufacturer's protocol with $\mathrm{DAB}$ in substrate buffer as chromogen (Thermo Scientific, Fremont, CA). For immunofluorescence studies, the above procedure was followed up until the peroxidase blocking step, at which point blocking was done for an hour in $12.5 \%$ bovine serum albumin (BSA) in PBS. Primary antibodies were incubated overnight in $12.5 \%$ BSA in PBS, and the next day, after washing in PBS, slides were incubated for $1 \mathrm{hr}$ in the dark with Alexa Fluor (Molecular Probes, Life Technologies, Grand Island, NY) secondary antibodies (1:100 in blocking solution). Following PBS wash, slides were mounted in DAPI-cotaining Vector medium. Primary antibodies used included Krt 8/18 (Fitzgerald), Krt 14 (Dako), Foxa1, AR, GFP, p63 (Santa Cruz), ${ }^{8,21,22}$ and Foxc1 (Sigma; http://www.proteinatlas.org/). Ki67 staining was performed by the Vanderbilt Experimental Pathology Core, as previously reported. ${ }^{8}$ Slides were visualized on a Zeiss Axio Imager.M1 microscope with Axiovision image capture software (Carl Zeiss Microimaging, Thornwood, NY).

\section{Quantitative RT-PCR}

RNA was extracted using the RNeasy kit from Qiagen (Valencia, CA, USA). Reverse transcription was conducted using SuperScriptII from Invitrogen (Carlsbad, CA, USA). Real-time PCR was performed on iCycler using iQ SYBR Green Supermix from Bio-Rad (Hercules, CA, USA). PCR was performed as follows: $95^{\circ} \mathrm{C}$ for $4 \mathrm{~min}$, followed by 40 cycles of $95^{\circ} \mathrm{C} / 30 \mathrm{~s}, 58^{\circ} \mathrm{C} / 30 \mathrm{~s}$, and $72^{\circ} \mathrm{C} / 30 \mathrm{~s}$. All samples were normalized to GAPDH. Results were expressed as fold change of each sample versus control using the $2^{(-\Delta \Delta C t)}$ method. ${ }^{23}$ Primer sequences used in this study are as follows: Foxc1 (5'-CCAATGCTTCCTTAAGCGGC-3',$\quad 5^{\prime}$-TGGCTAAGGCG GCCAAATAA-3'); Svs3b (5'-GGATGTGCTACTGTCCAAGA G-3' ${ }^{\prime}, 5^{\prime}$-TGCAAGGTGTTAAGGACTACAGG-3'); Svs6 (5'-C CACCAGCTTCTTTCTCCTTACA-3'， 5'-CCCATCTTCACCA TTGACCAT- $\left.3^{\prime}\right)$; Fam $3 \mathrm{~b}$ (5'-AAGGGCTTTGAGCTCCCTT C-3', 5'-CCTTCGATCTGGATCTCCGC-3'); Trpv6 (5'-ACCA GCCTTCCACCCCAT- $3^{\prime}, \quad 5^{\prime}$-TCAGAGCCTGGACATCGTT T-3'); Prom2 (5'-CGGGCTGGAGAAAATCCACT-3' ${ }^{\prime}, 5^{\prime}$-GCT TCGAAGTTCTCTGGCCT- $\left.{ }^{\prime}\right)$ Usp18 (5'-GACAGTCGACAG AAAGCCGT-3' , 5'-CAAATCAGGGACTCCTGCGT-3').

\section{Transient Transfection and Coimmunoprecipitation}

Coimmunoprecipitation of both endogenously expressed Foxc1 and transiently overexpressed Foxc1 with AR was 
performed, using the prostate myofibroblast cell line WPMY$\mathrm{AR}$, which was previously engineered to stably overexpress AR. ${ }^{24}$ Transient transfection of WPMY-AR cells with Foxc1 expression plasmid was performed using lipofectamine 2000 (Invitrogen) according to the manufacturer's recommendation, and essentially as previously reported. ${ }^{25}$ Transfected WPMY-AR cells expressing Foxc1 were washed three times with cold PBS and lysed with $1 \mathrm{ml}$ of nondenaturing lysis buffer $(50 \mathrm{mM}$ Tris, $150 \mathrm{mM} \mathrm{NaCl}, 10 \mathrm{mM}$ EDTA, $0.02 \%$ $\mathrm{NaN}_{3}, 50 \mathrm{mM} \mathrm{NaF}, 1 \mathrm{mM} \mathrm{Na}_{3} \mathrm{VO}_{4}, 1 \%$ NP-40, $1 \mathrm{mM}$ PMSF, $0.5 \mathrm{mM}$ dithiothreitol, and $1 \times$ concentration of complete protease inhibitor cocktail (Roche, Nutley, NJ). After sonication, centrifugation, and pre-clearing, $1 \mathrm{mg}$ of total cell lysate for each reaction was incubated at $4{ }^{\circ} \mathrm{C}$ overnight with $20 \mu \mathrm{l}$ (dry volume) protein G-Sepharose beads (Amersham Biotech, Piscataway, NJ), which were conjugated with $1 \mu \mathrm{g}$ experimental antibody or IgG antibody. Following overnight incubation, samples were centrifuged and the pelleted protein G-Sepharose beads were washed four times with lysis buffer and once with PBS followed by protein dissociation and western blotting analysis. The affinity-purified rabbit polyclonal antibody (HPA040670, Sigma-Aldrich) was used to immunoprecipitate Foxc1. To identify interactions between Foxcl and AR western blotting was subsequently performed with an anti-AR (N20; Santa Cruz Biotechnology, Santa Cruz, CA). IgG was used as a negative control.

\section{Microarray Analysis}

Microarray analysis on RNA extracted from anterior prostates (AP) dissected from control and experimental mice, as well as the seminal vesicles dissected from control mice, was performed using the GeneTitan1.1.ST mouse array (Affymetrix Corp; Santa Clara, CA) by the Vanderbilt Technologies for Advanced Genomics (VANTAGE) core. The GeneTitan1.1.ST mouse array chip contains probes for 26166 coding transcripts (RefSeq), and $\sim 7000$ non-coding transcripts, including 2000 long intergenic non-coding transcripts. Instrument control and data acquisition were performed using the Affymetrix GeneChip Command Console. Fold change was calculated in a log base 2 space, using the following formula (Fold Change $=2^{\text {(experimental }- \text { control) }}$ ), followed by inverting and negating ratios less than 1. Microarray analysis was performed both to identify increases in seminal vesicle-associated gene expression in the AP of Foxa1 KO mice and to identify genes whose expression was altered coordinately following both castration and Foxa1 KO. See Supplementary Data for gene lists.

\section{RESULTS}

Foxa1 Expression is Restricted to Luminal Epithelium: a Subpopulation of Cells that are Efficiently Targeted by PBCre4

As FOXA1 expression in the human prostate is restricted to luminal epithelium, while basal prostate epithelium fails to exhibit positive FOXA1 immunostaining, ${ }^{26}$ we performed dual immunofluorescence microscopy to determine the extent of Foxal colocalization with cytokeratin (Krt) 8/18 and Krt14, which are markers of luminal and basal epithelium, respectively (Figure 1). We performed this staining on the anterior prostates (AP), as this lobe of the murine prostate has the largest population of Krt14-positive basal cells. As is the case in humans, ${ }^{26}$ results show that Foxal expression is restricted to Krt 8/18-positive epithelium (Figure 1d, inset) in the adult mouse and is not detected in the Krt14positive basal cell layer (Figure $1 \mathrm{~h}$, inset). Promoters vary in their penetrance, which is the primary determinant in their ability to efficiently drive transgene expression in specific cell types. Therefore, selecting the proper promoter to drive Cre recombinase is essential. Initial immunofluorescence studies required that we utilize a promoter specifically expressed in luminal epithelium to achieve genetic ablation of Foxal in the prostate. In order to verify the suitability of the PBCre4 transgenic line for the targeting of Foxa1positive luminal prostate epithelium, we bred $\mathrm{PBCre} 4$ mice

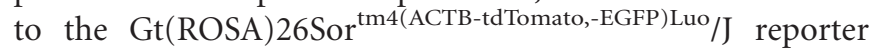
line (Figure 2). Dual immunofluorescence studies (2A; DAPI, 2B; Foxa1, 2C; GFP, 2D Merge) on the AP of an adult mouse revealed that PBCre4 targeted a subset of cytokeratin-8/ 18-positive luminal epithelium (Figure 2c). A range of GFP-positive cells in which Cre was activated was detected in the anterior prostate (AP), with the distal tips and base of the AP being the most affected. A similar range of GFPpositive cells in the lateral (LP), dorsal (DP), and ventral (VP) prostates were GFP positive (see Supplementary Data). Dual immunofluorescence for GFP and Foxal revealed that Foxa1-positive murine prostate epithelium was efficiently targeted by Cre recombinase (Figure 2d) in the adult AP. In summary, these data suggest $\mathrm{PBCre} 4$ is suitable to achieve conditional Foxal knockout in the murine prostatic luminal cells.

\section{Prostate-Specific Mosaic Foxa1 Knockout Results in Florid Hyperplasia}

To determine the impact of Foxal ablation on tissue morphology, PBCre4/Foxal $1^{\text {loxp }}$ and PBCre4/Foxal ${ }^{\text {loxp/loxp }}$ mice (and control Foxal $1^{\text {loxp }}$ and Foxa $1^{\text {loxp/loxp }}$ mice) were aged and killed at 7, 24, and 33 weeks of age. Histological analysis (Figure 3; top panel Foxal ${ }^{\text {loxp/loxp }}$ control mice; bottom panel PBCre4/Foxal ${ }^{\text {loxp/loxp }}$ mice) showed changes as early as 7 weeks of age in the AP and DP, which progressed to florid hyperplasia complete with cribriform patterning (Figure 3; bottom panel). Progressive hyperplasia was present in all lobes by 24 weeks. We did not observe epithelial cell invasion into the prostatic stroma. In keeping with PBCre4/

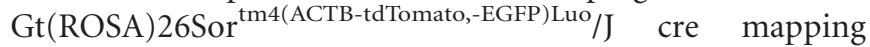
studies, Foxal knockout was mosaic in nature in all lobes of the prostate (Figure 4a; top left). Ki67 staining revealed a increase in the number of proliferating cells (Figure $4 \mathrm{~b}$; top right), with the largest proportion of Ki67 staining present in the AP and DP. We previously reported that FOXA1 loss 
coincided with increased Ki67 staining in human bladder cancer specimens, suggesting that Foxa1-negative murine prostate epithelium would be Ki67 positive. ${ }^{21}$ However, this did not appear to be the case, as increases in Ki67 labeling were not restricted to Foxa1-negative cells as shown by dual immunofluorescence of the AP dissected from 24 week-old PBCre4/Foxa1 loxp/loxp mice (Figure 4c; bottom panel). These results indicate PBCre4-mediated Foxal knockout increases the proliferation of prostate epithelium in a manner that is not simply restricted to Foxal knockout epithelium.

\section{PBCre4/Foxa $1^{\text {loxp/loxp }}$ Mice Exhibit Altered Localization of p63-Positive Basal Cells}

Embryonic Foxal knockout was previously reported to result in an expansion of the basal cell population. ${ }^{8}$ Immunohistochemistry for p63 in 24 week old PBCre4/Foxa $1^{\text {loxp/loxp }}$ mice revealed a similar increase in the number of basal epithelial cells (Figure 5a). In addition to being expanded, the p63-positive cell population was not limited to the basal compartment of the epithelium (see PBCre4/Foxal loxp/loxp Figure 5a). We performed dual immunofluorescence on the AP of 24 week old PBCre4/Foxal $1^{\text {loxp/loxp }}$ mice to determine whether cells with PBCre4-mediated recombination and Foxa1 knockout were also activating p63 expression. Dual immunofluorescence clearly showed p63 is not expressed in Foxa1-null luminal epithelium (Figure 5b). Increases in p63 could also be mediated by an expansion of the intermediate cell population, indicating a failure of Foxal negative cells to fully differentiate. Dual immunofluorescence of 24 week old PBCre4/Foxal loxp/loxp mice demonstrated that Krt8/18 and p63 expressions were mutually exclusive in the AP, indicating the lack of an expanding intermediary cell population in response to Foxal knockout (Figure 5c).

\section{While Foxa1 Knockout Prostates Retain Androgen Sensitivity, Foxa1-Positive Cells Appear More Sensitive to Castration}

Examination of the weights of anterior prostates dissected from the intact Foxal $1^{\text {loxp/loxp }}$ and PBCre4/Foxal $1^{\text {loxp/loxp }}$ mice

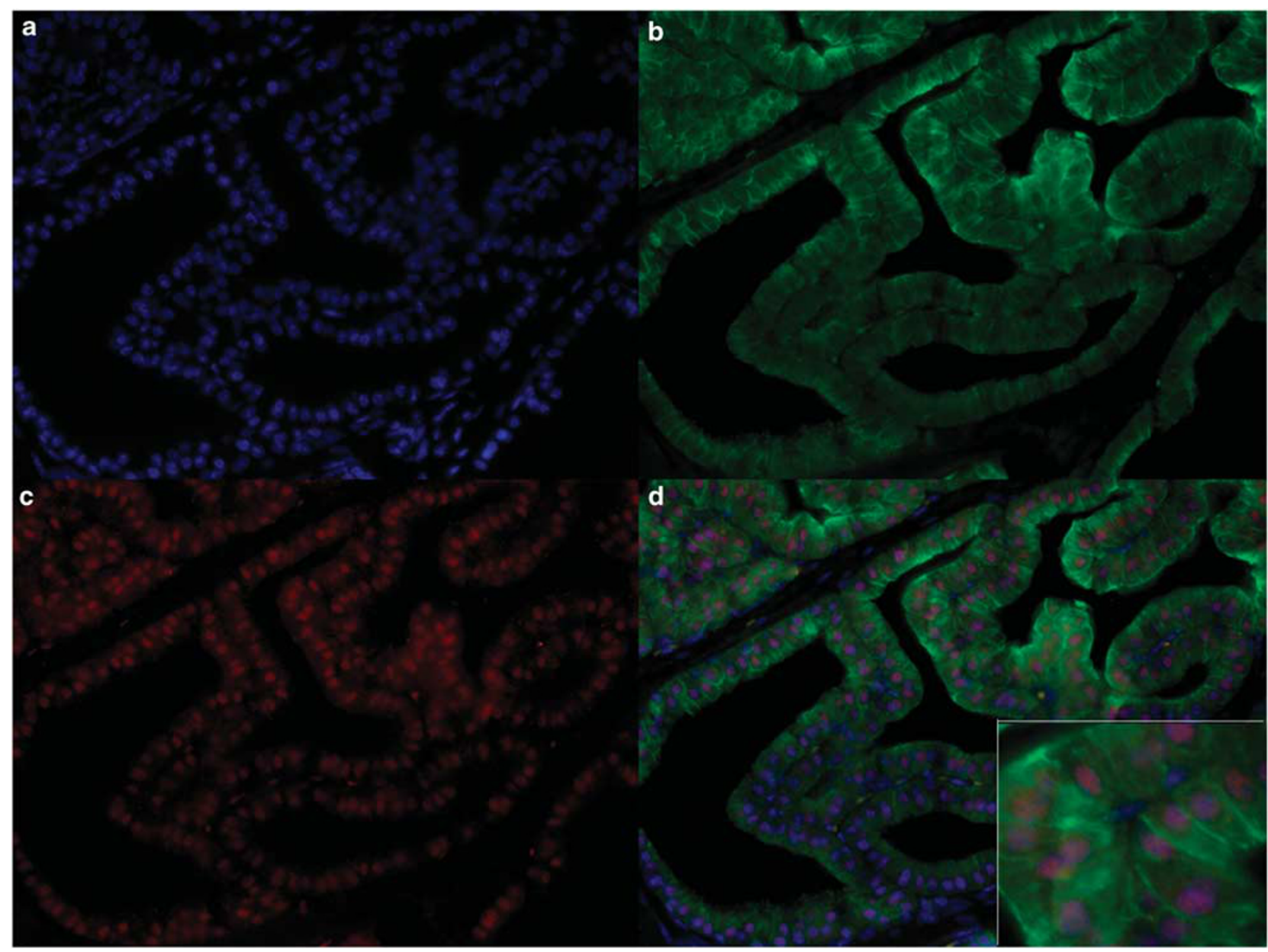

Figure 1 Foxa1 expression in murine prostate epithelium co-localizes with cytokeratin 8/18 expression, but is absent in cytokeratin 14-positive basal prostate epithelium. Panels a (DAPI), b (Cytokeratin 8/18), c (Foxa1), and d (merge) show co-localization of Foxa1 with cytokeratin 8/18-positive prostate epithelium in the anterior prostate. Panels e (DAPI), $\mathbf{f}$ (cytokeratin 14), $\mathbf{g}$ (Foxa1), and $\mathbf{h}$ (merge) show cytokeratin 14-positive basal epithelium does not express Foxa1 in the mouse prostate anterior prostate (see annotation arrows in $\mathbf{h}$ inset). 


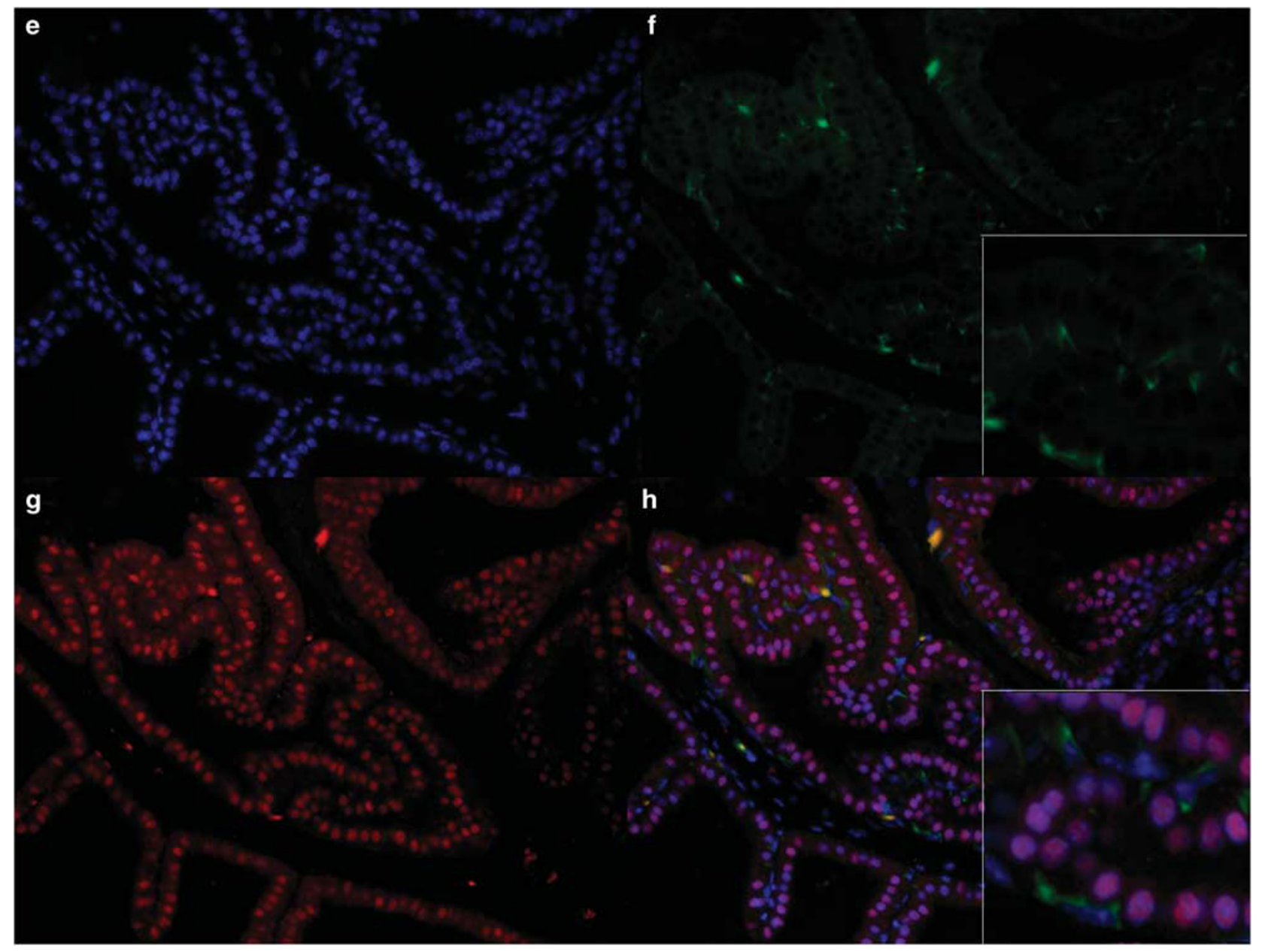

Figure 1 Continued.

revealed significant differences $(P=0.013)$, which may be attributed to decreased secretory activity of Foxal KO prostates. ${ }^{8}$ PBCre $4 / F o x a 1^{\text {loxp/loxp }}$ mice retain AR expression (see Supplementary Data), but it was unclear how Foxa1 knockout would influence prostate regression following castration. We therefore examined the prostates of 33-week-old PBCre4/ Foxal $1^{\text {loxp/loxp }}$ mice and control Foxal $1^{\text {loxp }}$ and Foxal $1^{\text {loxp/loxp }}$ mice 2 weeks after castration. Both Foxal loxp/loxp and PBCre4/Foxa1 ${ }^{\text {loxp/loxp }}$ mice showed prostatic atrophy after castration, as indicated by significant decreases in prostate weight (Figure 6a; Student's $t$-test; $P<0.0005$ ), decreased Ki67 immuno positivity, as well as histological alterations (regression of glandular structure, increased expansion of smooth muscle, and altered nuclear to cytoplasmic ratio; data not shown). Comparison of tissue dissected from intact and castrated 33-week-old PBCre4/Foxa1 $1^{\text {loxp/loxp }}$ mice revealed a significant decrease in the number of Foxa1-positive cells following castration. (Figure 6c; Mann-Whitney test; $P=0.0003)$. No significant changes in Foxal immunostaining were detected when comparing intact and castrated tissue dissected from 33-week-old Foxal loxp/loxp mice
(Figure 6c; Mann-Whitney test; $P=0.7$ ). These results potentially indicate that the subpopulation of prostate luminal epithelium not targeted by PBCre4, and therefore retain Foxa1, are especially sensitive to androgen deprivation, while the Foxa1-null cells are more resistant to castration.

\section{PBCre4-Foxa1 KO Prostates Express Markers of Seminal Vesicle Differentiation}

We performed microarray analysis to gain further insights into changes in gene expression following Foxal knockout. Microarray analysis on the Foxal KO AP dissected from a 33-week-old mouse, as well as age-matched controls, revealed that three of the six major seminal vesicle secretions (SVS) genes were upregulated in this lobe of the murine prostate. Therefore, microarray analysis was performed on the seminal vesicles, and a comparison of genes expressed in intact seminal vesicles was made to the Foxa1 KO AP. This analysis revealed that 57 genes normally specific to the seminal vesicles were now expressed by the Foxa1 KO AP (see Supplementary Data for gene list). Because Foxal activity is so 


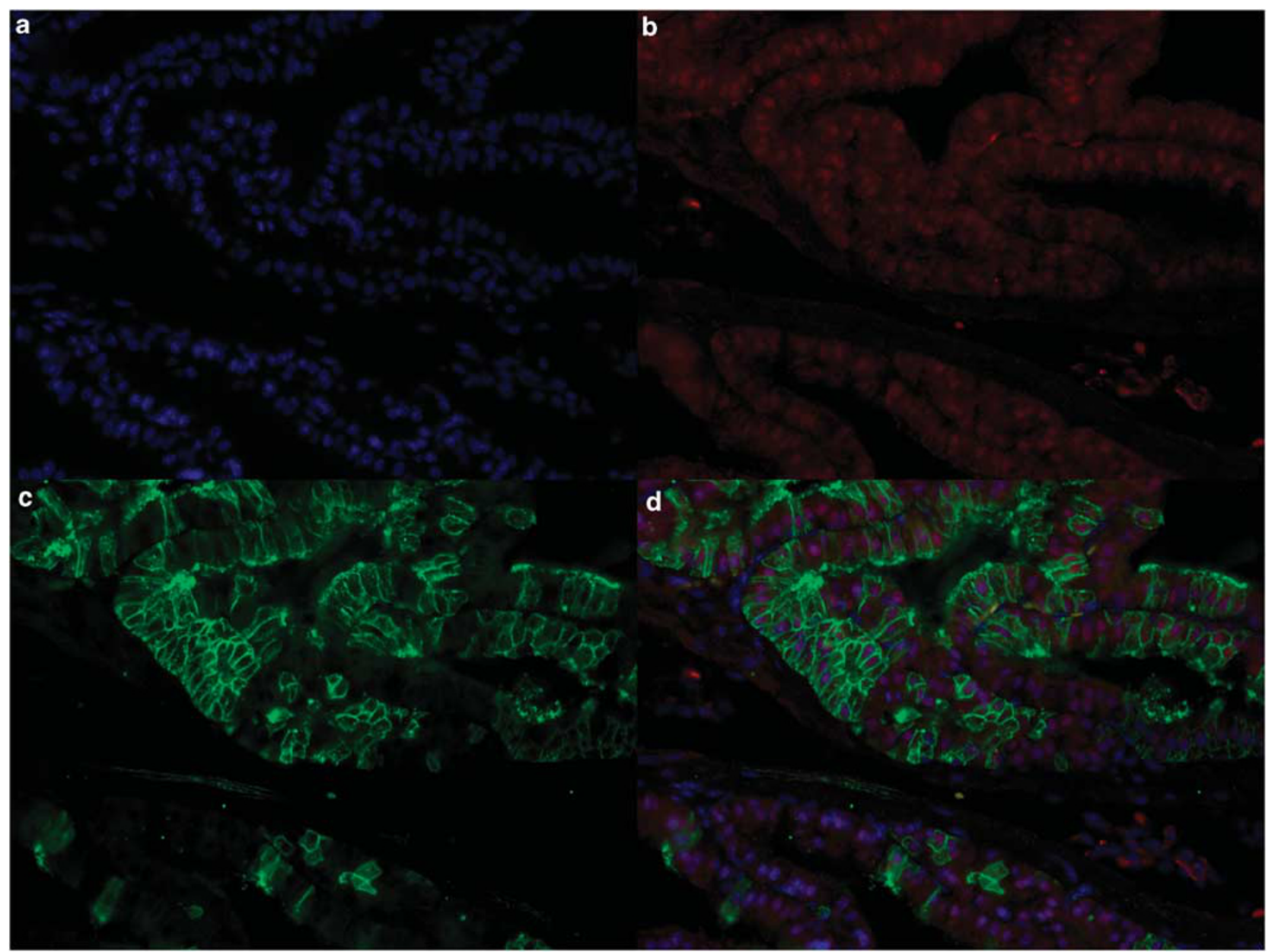

Figure 2 PBCre4 efficiently targets Foxa1-positive prostate epithelium. Immunofluorescence on anterior prostate tissue dissected from PBCre4/

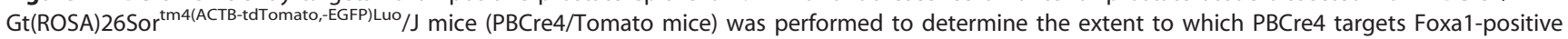
prostate epithelium. The presence of membrane bound GFP in PBCre4/Tomato mice is indicative of a cre-mediated recombination event. Panels a (DAPI), b (Foxa1), c (GFP), and $\mathbf{d}$ (merge) show that Foxa1-positive prostate epithelium in the anterior prostate are efficiently targeted by PBCre4 in the AP. In terms of percentage, a range of GFP-positive cells where Cre was activated was detected in the anterior prostate (AP), with the distal tips and base of the AP being the most affected (see Supplementary Data for analysis of other prostate lobes).

intricately tied to AR function in the prostate, Foxal KO could alter pathways that are associated with response to circulating androgens. Therefore, we performed microarray analysis to identify the number of genes (if any) that were coordinately regulated by both castration and Foxal KO (see Supplementary Data). This analysis revealed that, of 240 genes whose expression decreased in response to castration, $47(19.6 \%)$ of the same genes changed following Foxa1 KO. Of 271 genes whose expression increased following castration of a control prostate, $30(11.1 \%)$ of the same genes changed following Foxa1 KO.

In regard to the increased expression of genes associated with seminal vesicle, qRT-PCR and subsequent statistical analysis of cDNA samples prepared from AP dissected from 33-week-old Foxal loxp/loxp mice and aged-matched PBCre4/ Foxal ${ }^{\operatorname{loxp} / \text { loxp }}$ mice revealed significant increases (Student's $t$-test) in the expression of Foxc1, as well as the seminal vesicle marker genes Svs3b and Svs6 (Figure 7a;). In addition, we were able to detect significant increases in the expression of Fam3b, Trpv6, and Prom2, all of which are normally expressed in a relatively seminal vesicle-specific manner (Figure 7a). Immunohistochemistry of murine seminal vesicle showed that Foxc1 is expressed at high levels in this androgen target tissue (Figure 7b; left panel). Furthermore, while we failed to detect Foxcl expression in AP prostates dissected from 40-week-old Foxa ${ }^{\text {loxp/loxp }}$ (Figure 7b; middle panel), we detected increased Foxcl expression in AP epithelial cells dissected from age-matched PBCre4/Foxa $1^{\text {loxp/loxp }}$ mice (Figure 7b; right panel). As we have previously shown that Foxal interacts with the AR in human prostate epithelial cells, we postulated that Foxc1 may also physically interact with AR in tissues where these transcription factors are coexpressed. To test this hypothesis, we performed coimmunoprecipitation experiments on cells expressing both AR and 


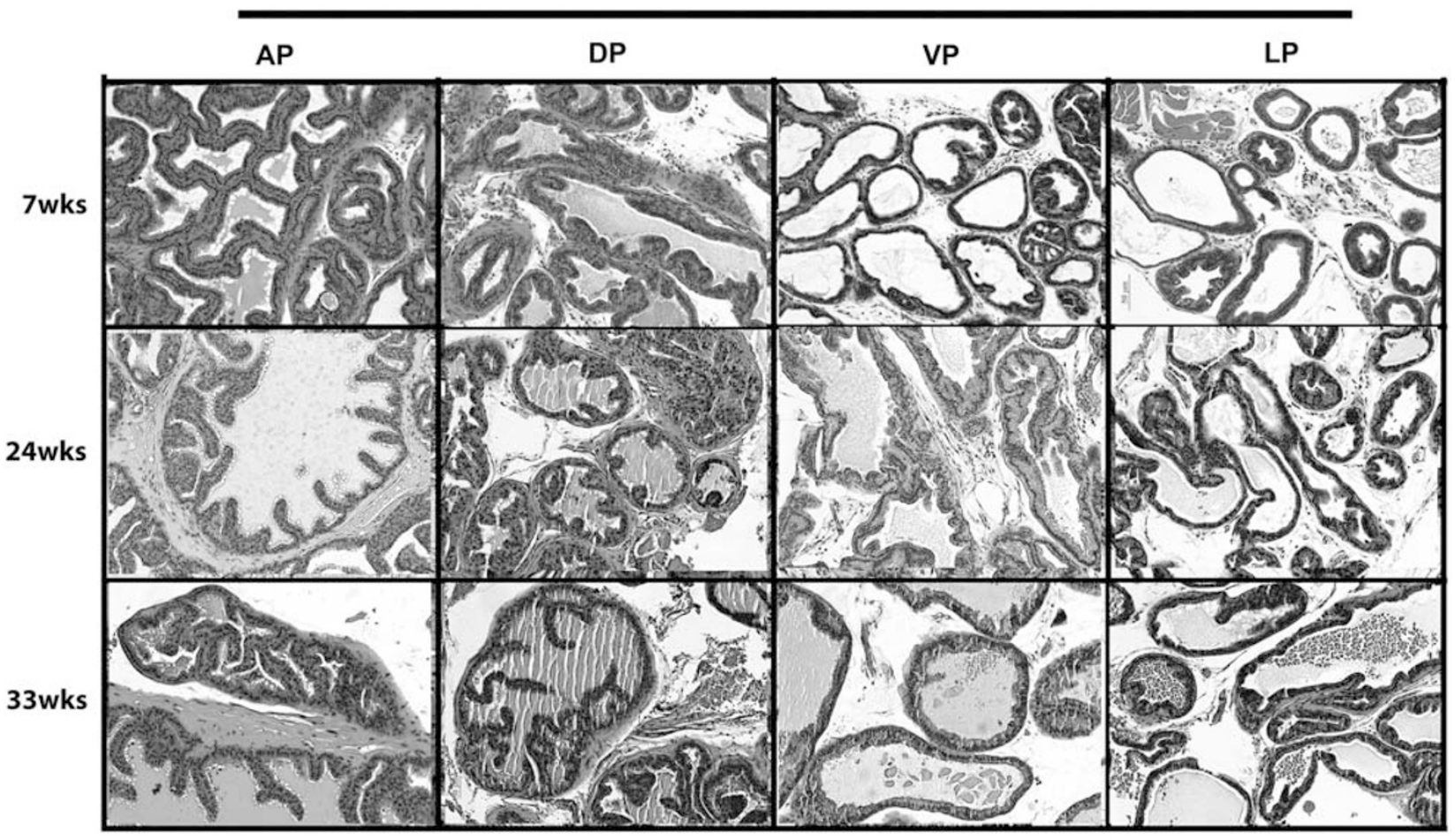

b PBCre4/Foxa1

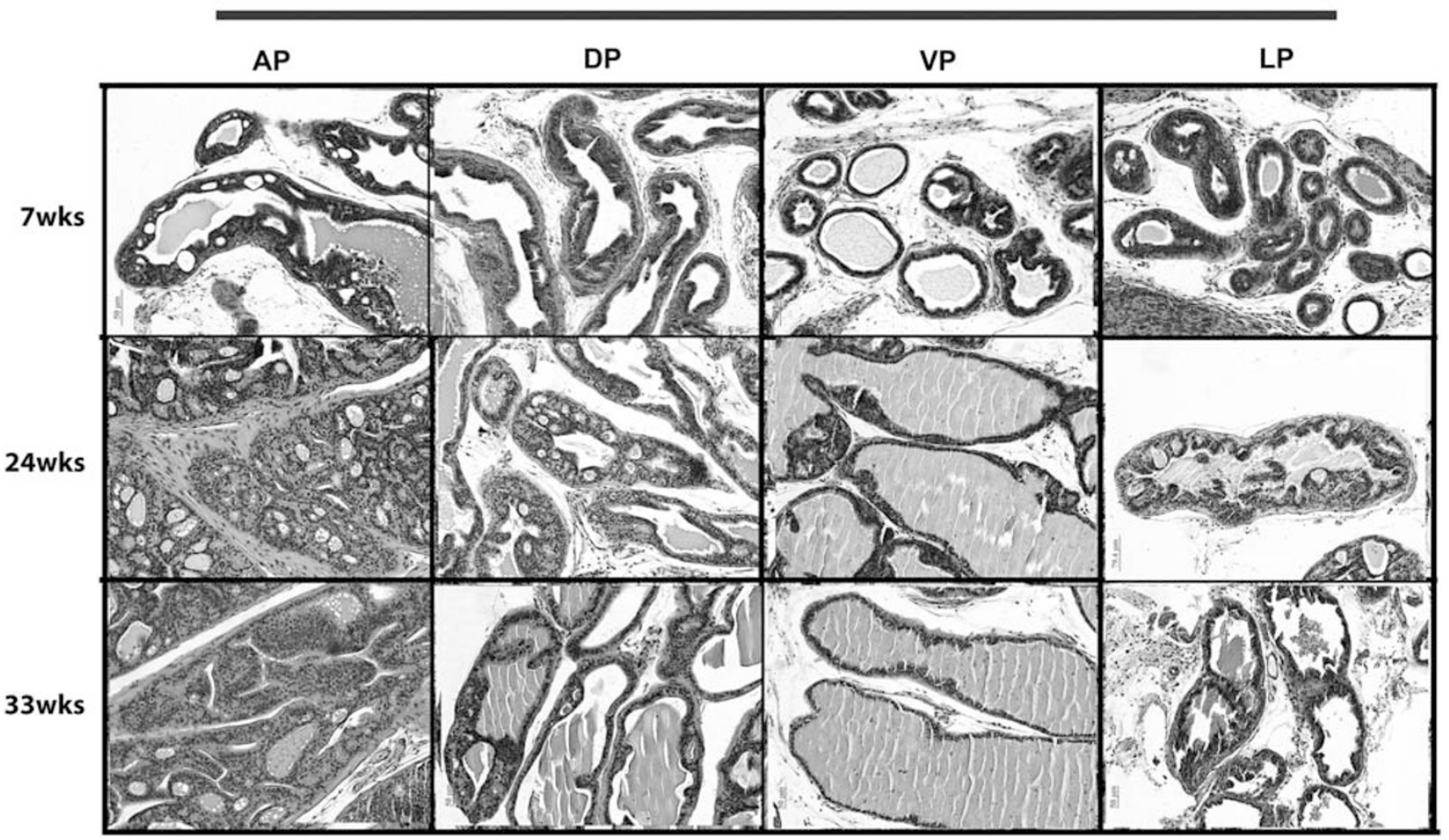

Figure 3 Prostates dissected from PBCre4/Foxa1 $1^{\text {loxp/loxp }}$ mice exhibit progressive hyperplasia with cribriform patterning. Anterior, dorsal, ventral, and lateral prostatic tissue dissected from 7-, 24-, and 33-week-old Foxa1 ${ }^{\text {loxp/loxp }}$ control mice, which exhibit normal histomorphology following H\&E stain (a). Conversely, H\&E stain of anterior, dorsal, ventral, and lateral prostatic tissue dissected from age-matched PBCre4/Foxa1 ${ }^{\text {loxp/loxp }}$ mice reveals largely progressive hyperplasia with extensive cribriform patterning (b). This phenotype was most pronounced in the anterior prostate. 
Foxc1. The WPMY-AR cell line, which is a myofibroblastic cell line engineered to stably overexpress $\mathrm{AR},{ }^{24}$ was chosen because Foxcl expression was previously reported to be increased in prostatic stroma. ${ }^{27}$ Immunoprecipitation of Foxc1, followed by western blotting analysis, showed that $\mathrm{AR}$ co-precipitated indicating a physical interaction with endogenously expressed Foxc1 in WPMY-AR cells (Figure 7c). Our ability to detect this interaction was enhanced following overexpression of Foxc1 in WPMY-AR cells (Figure 7d). These results indicate Foxc1 interacts with $\mathrm{AR}$, and that this complex may be important in the regulation of AR target genes in Foxal-negative cells.

\section{DISCUSSION}

We had previously reported an essential role for Foxal expression during normal prostate development, ${ }^{8}$ where knockout of Foxal during embryonic development resulted in the failure of prostate morphogenesis, an expansion of a p63-positive basal cell population, and the retention of embryonic markers of prostate development, including Foxa2. However, embryonic knockout of Foxa1 made it impossible to determine the importance of this transcription factor for the maintenance of normal prostatic differentiation that occurs with sexual maturation. In the current study, we used the PBCre4 system to ablate Foxal in a prostate-specific manner during sexual maturation. We show that the PBCre4 efficiently targets the luminal prostate epithelium that expresses Foxa1 (see Figures 1 and 2, as well as Supplementary Data), and that PBCre4-mediated Foxal knockout results in progressive and florid hyperplasia with cribriform patterning, which is most pronounced in the AP (see Figure 3). These studies revealed an important role for Foxal Foxa1 ${ }^{\text {loxp/loxp }}$

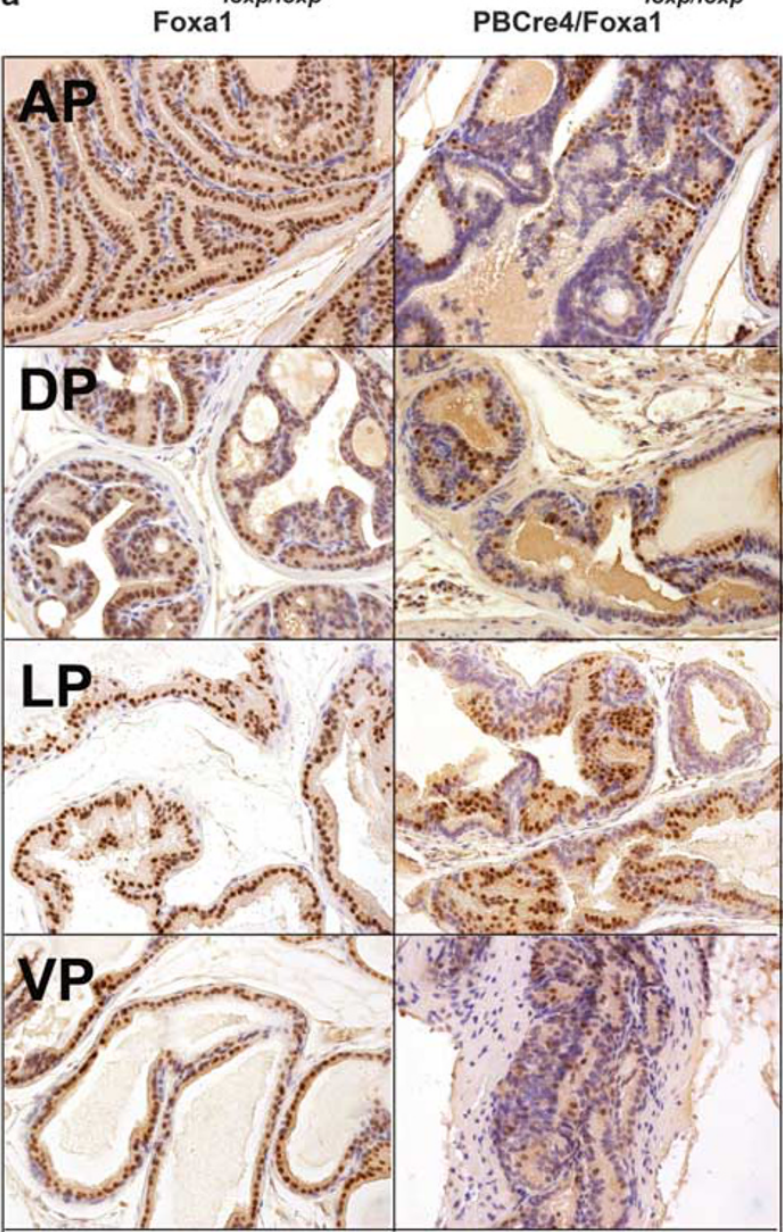

b Foxa1 ${ }^{\text {loxp/loxp }}$ PBCre4/Foxa1

loxp/loxp

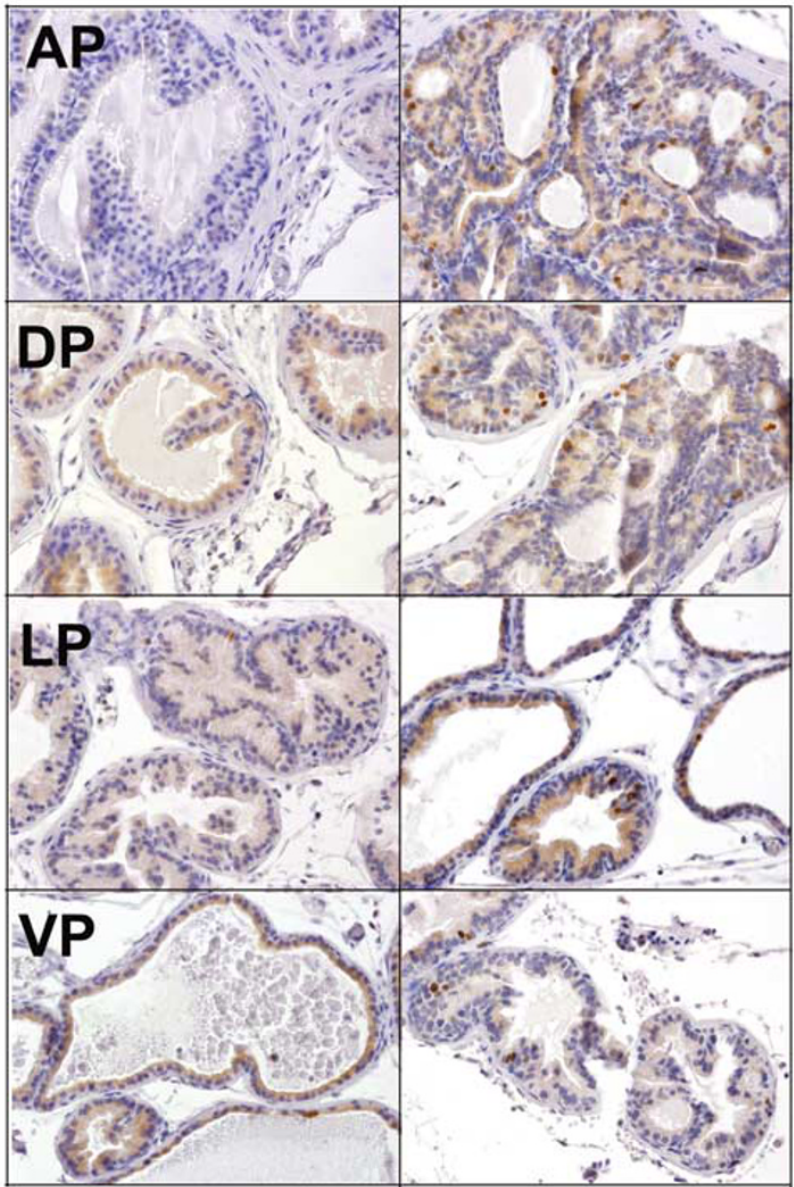

Figure 4 PBCre4-mediated Foxa1 knockout is mosaic in nature and results in increased epithelial proliferation. Panel a: Foxa1 immunostaining of anterior, dorsal, lateral, and ventral prostate tissue dissected from 24-week-old control Foxa $1^{\text {loxp/loxp }}$ mice and age-matched PBCre4/Foxa1 ${ }^{\text {loxp/loxp }}$ mice reveal Foxa1 knockout is mosaic in nature, in keeping with PBCre4/Tomato mice cre mapping studies. Panel b: Ki67 staining reveals an increase in the number of proliferating cells following Foxa1 knockout in 24-week-old PBCre4/Foxa1 loxp/loxp mice (see Supplementary Data for graphical representation of Ki67 index). Panel c: dual immunofluorescence for Foxa1 (red) and Ki67 (green) shows that detection of Ki67 staining is not limited to Foxa1-negative cells in the anterior prostate (see merged panel in c). White arrows point to Foxa1-negative, Ki67-positive epithelium, while yellow arrows point to Foxa1-negative epithelium that is Ki67 positive. 
C

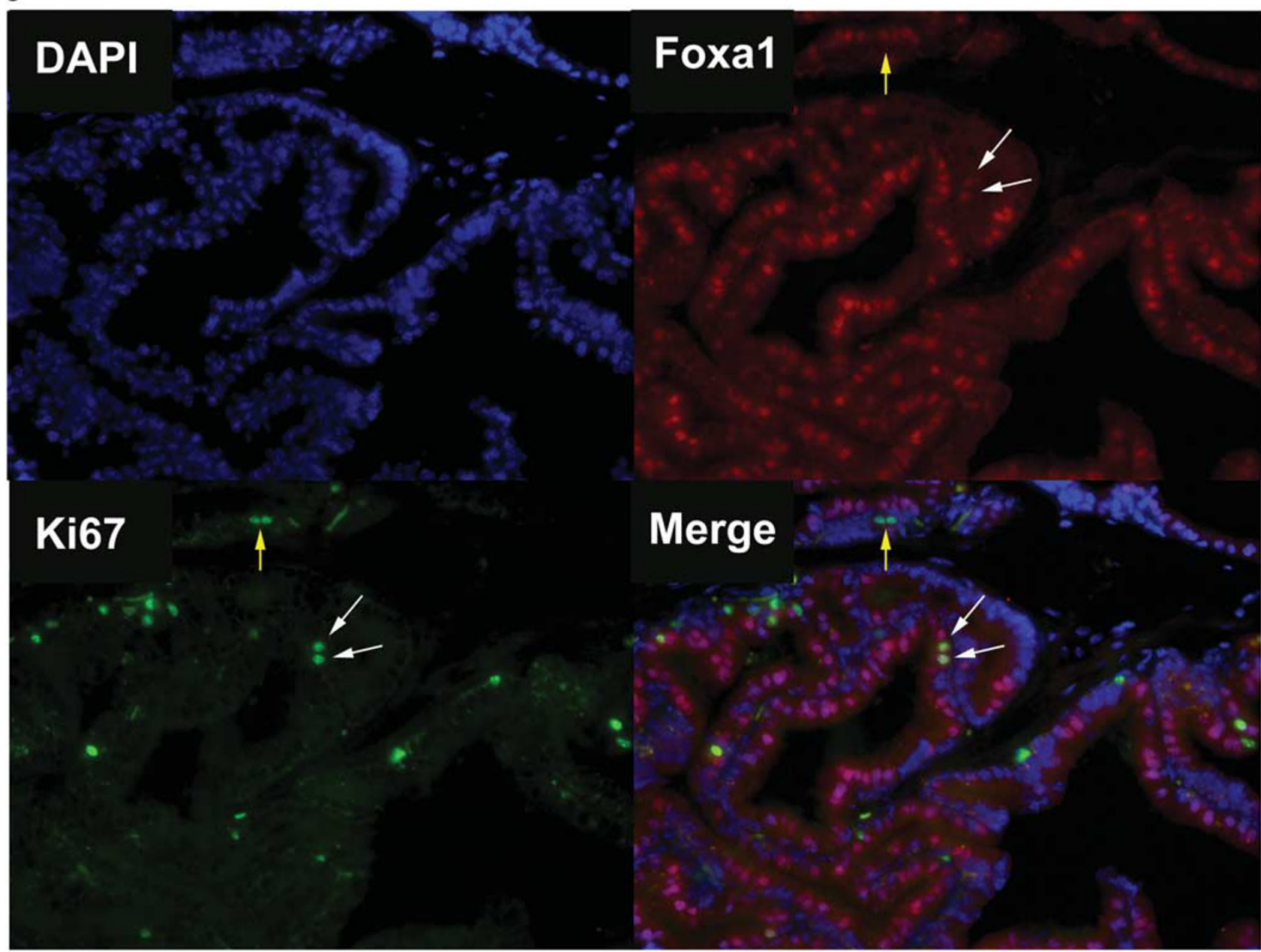

Figure 4 Continued.

expression in the adult prostate for the maintenance of a differentiated phenotype.

PBCre4 targets luminal prostate epithelium, resulting in a 'mosaic' knockout of Foxa1. This phenomenon revealed differences in the relative androgen sensitivity of Foxa1positive and -negative cells. These results indicate that Foxa1positive cells are more sensitive to castration (or that Foxal negative cells are more resistant to castration), enabling a relative expansion of the Foxa1-negative epithelial cell population (Figure 6). It is impossible to know whether these results are a direct influence of decreased AR action on the epithelial cell compartment (which is now heterogenous in regard to Foxal expression) or whether they are a reflection of differences in which FOXA1-positive and -negative epithelium responds to androgen-regulated paracrine signals from the stroma. ${ }^{28}$ Put differently, in the epithelium our observation may be the result of the physical interaction between Foxal and AR, as well as the fact that these transcription factors act in a cooperative manner to regulate gene expression in response to paracrine stromal signals.
Further studies are planned to identify the exact mechanism by which Foxal status influences sensitivity to androgens. It is also interesting to note that PBCre4 induced recombination in the majority of prostate epithelium as revealed by our reporter mouse studies (see Figure 2 and Supplementary Data), which would predict a more complete knockout of Foxal then was revealed in our studies (see Figure 4). This apparent discrepancy may indicate that Foxa1 knockout is initially toxic in a subset of prostate epithelium, while additional populations in which Foxal is successfully ablated survive this genetic change. Nonetheless, Foxa1 ablation with the PBCre4 did result in a striking phenotype (see Figure 3). Further study is required to determine the short-term impact of Foxal knockout.

AR expression is widely distributed among tissues derived from the developing germ layers (endoderm, ectoderm, and mesoderm); yet, precise genes associated with differentiation are regulated by $\mathrm{AR}$ in specific cell populations. ${ }^{29}$ The cell-specific expression of AR target genes is explained via the combinational control of gene expression model. This 
a

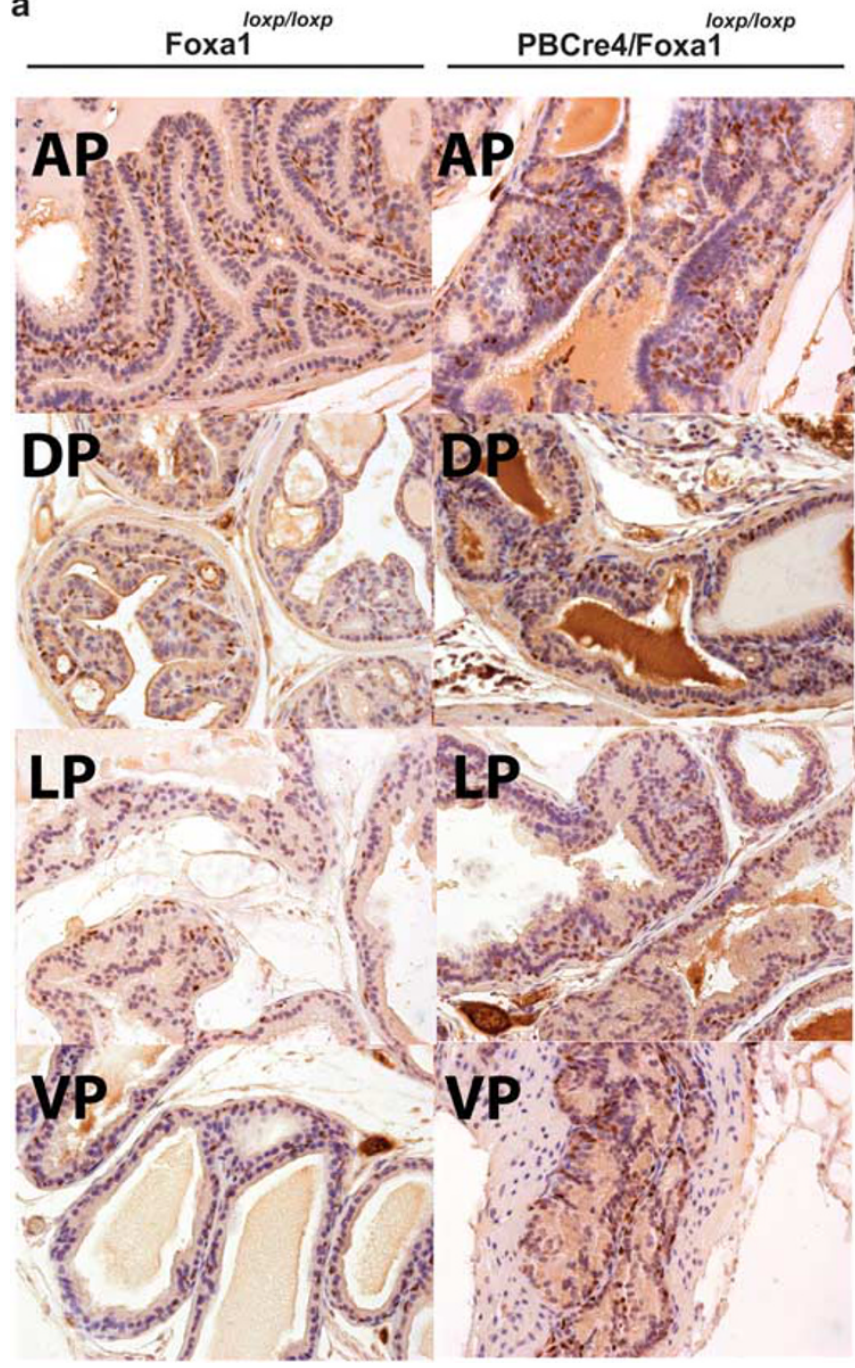

b

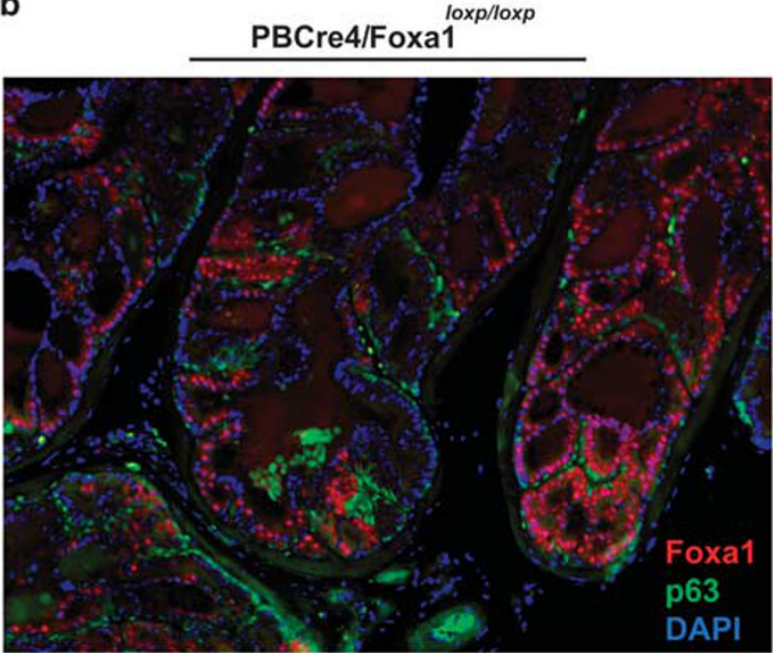

C

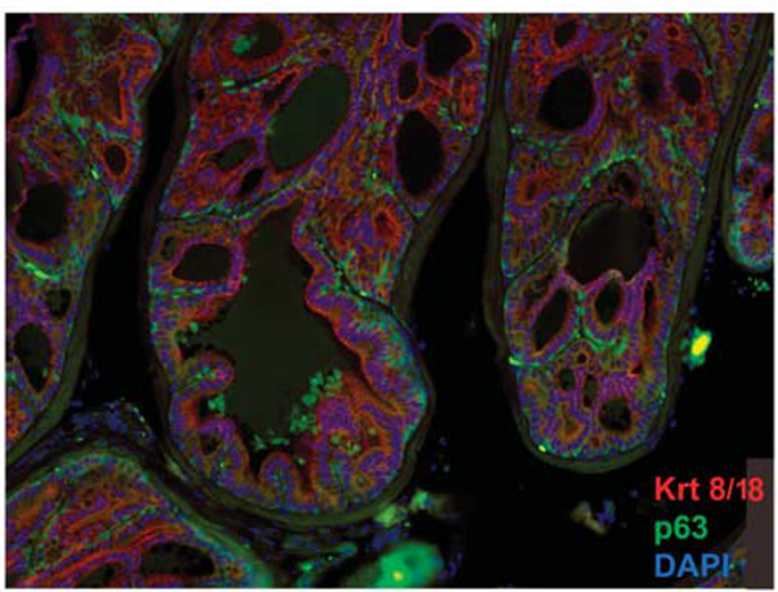

Figure 5 Foxa1 knockout results in a non-cell autonomous expansion of p63-positive basal epithelium. (a) Immunostaining for the basal cell marker p63 in prostate tissue dissected from 24 -week-old control Foxa $1^{\text {loxp/loxp }}$ mice reveals a normal staining pattern limited to basal epithelium adjacent to the prostatic stroma. Immunostaining for p63 in age-matched PBCre4/Foxal loxp/loxp mice reveals an expansion of the basal cell population. In addition, p63-positive epithelium in PBCre4/Foxa $1^{\text {loxp/loxp }}$ mice is not limited to the basal cell population and are is throughout the prostatic epithelium. (b) Dual immunofluorescence for Foxa1 (red) and p63 (green) reveals that Foxa1-negative prostate epithelium exhibits a lack of p63 expression, indicating that absence of Foxa1 does not increase p63 expression in a cell autonomous manner. (c) Intermediate prostate epithelial cells are a rare population typified by dual positivity for the luminal markers cytokeratin $8 / 18$ and the basal marker p63. In order to determine whether increases in the number of p63 positive cells following Foxa1 knockout is the result of an increase in the number of intermediate prostate cells, we performed dual immunofluorescence for cytokeratin 8/18 (red) and p63 (green). Results show a mutual exclusivity between cytokeratin 8/18 and p63 staining, indicating the absence of an increased population of intermediary prostate epithelial cells in PBCre4/Foxa $1^{\text {loxp/loxp }}$ mice.

Figure 6 Castration increases the number of Foxa1-negative prostate epithelial cells in PBCre4/Foxa1 loxp/loxp mice. (a) Analysis of wet weights of the anterior prostates dissected from intact and castrated 33-week old Foxa $1^{\text {loxp/loxp }}$ and PBCre4/Foxa $1^{\text {loxp/loxp }}$ prostates revealed a significant decrease in weight following castration in both control and Foxa1 knockout mice. (b) Comparison of intact 33-week old Foxa $1^{\text {loxp/loxp }}$ and PBCre4/Foxa1 ${ }^{\text {loxp/loxp }}$ prostates revealed significant differences in weight, which is in keeping with reduced cytoplasm of Foxa1 negative epithelium (see annotation arrows in b), further suggesting a role for Foxa1 in the maintenance of a differentiated phenotype. (c) Inspection of H\&E staining from both Foxa ${ }^{\text {loxp/loxp }}$ and PBCre4/Foxa $1^{\text {loxp/loxp }}$ prostates revealed atrophy of the gland following castration in both groups indicative of androgen sensitivity and consistent with decreased prostatic weight following castration (H\&E stain). Although PBCre4-mediated Foxa1 knockout is mosaic in nature, castration results in a decrease in the number of Foxa1-positive cells in castrated PBCre4/Foxa1 1oxp/loxp prostates compared with control Foxa1 loxp/loxp mice (d) Graphical representation of the \% of Foxa1-positive cells in intact and castrated PBCre4/Foxa1 ${ }^{\text {loxp/loxp }}$ prostates. Comparison of intact and castrated Foxa1 loxp/loxp anterior prostates revealed no significant differences in the number of Foxa1-positive cells after castration in genetic controls (Mann-Whitney test; $P=0.7)$, whereas comparison of intact and castrated PBCre4/Foxa $1^{\text {loxp/loxp }}$ anterior prostates did reveal significant differences in the number of Foxa1 positive cells following castration in Foxa1 KO mice (Mann-Whitney test; $P=0.0003$ ). 
paradigm predicts that the presence and activity of a unique combination of different regulatory proteins during development and in adult tissues dictates cell fate. ${ }^{29,30}$ The family of forkhead proteins is particularly interesting as their expression is temporally restricted during development and specific to adult cell populations. We reported that the AR and Foxal protein formed a complex via their DNA-binding domains on multiple prostate specific genes and that this complex is critical for differentiation of the prostate gland. ${ }^{8,9,11}$ It is now widely recognized that the AR/FOXA1 complex is fundamental in androgen action in the prostate, and the function of FOXA1 has been extended to include
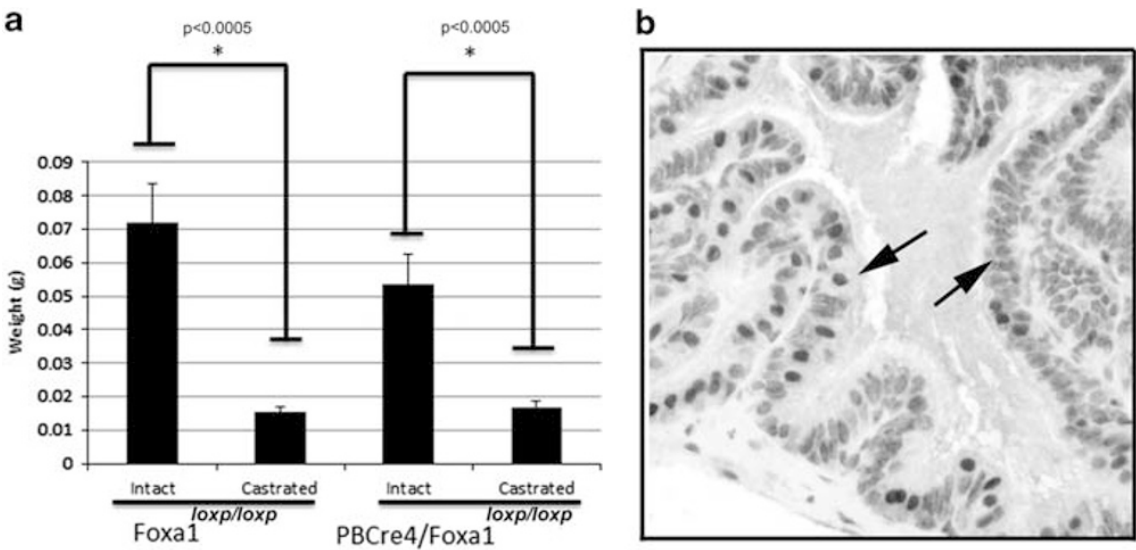

C

$$
\text { Foxa1 } 1 \text { (oxp/loxp }
$$

Foxa1
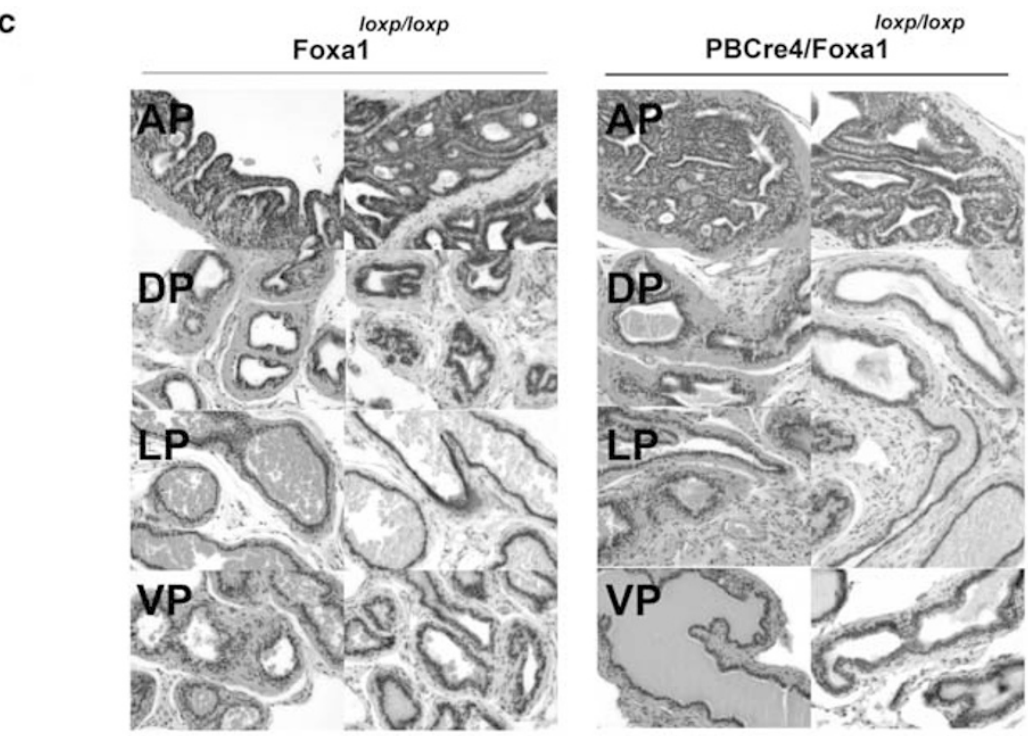

d

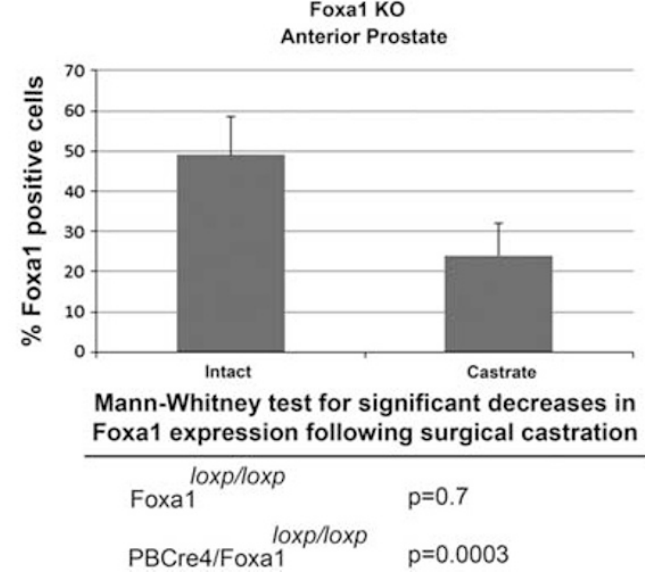

Figure 6 For caption see page 735. 
b

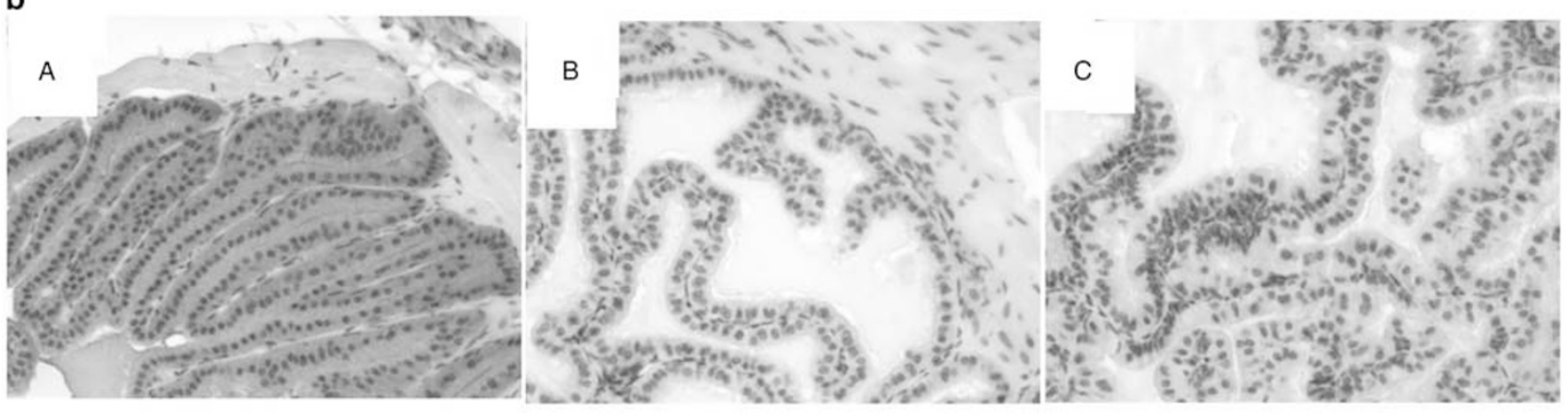

C

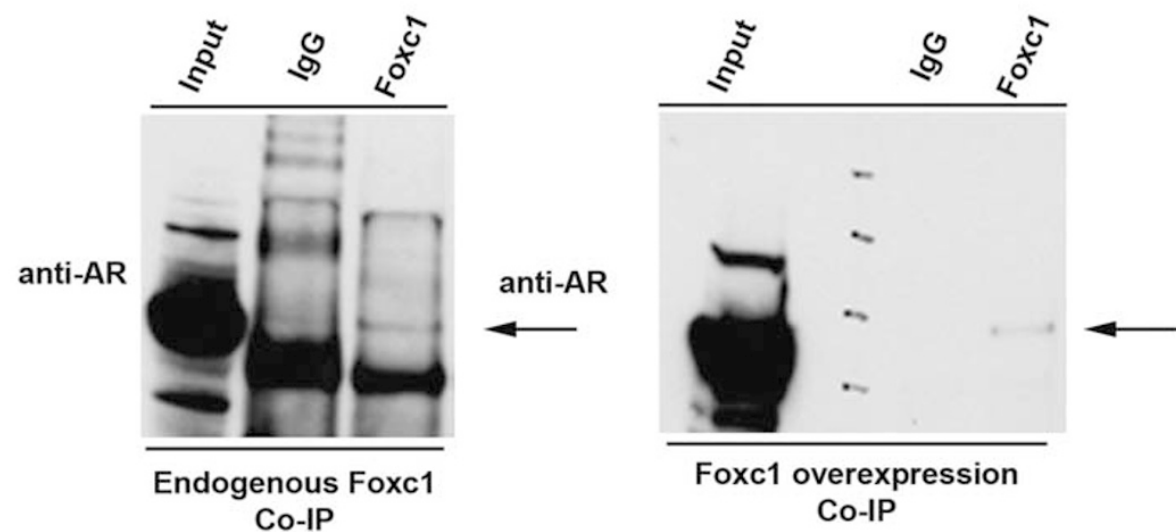

Figure 7 PBCre4-mediated Foxa1 knockout results in the increased expression of the novel AR-interacting protein Foxc1 and additional seminal vesicle-associated genes. (a) QRT-PCR analysis shows increases in the expression of Foxc1 and the seminal vesicle-associated genes Svs3b, Svs6, Fam3b, Trpv6, and Prom2. (b) Immunohistochemistry for Foxc1 in murine (left panel) seminal vesicle, (middle panel) Foxa ${ }^{\text {loxp/loxp }}$ anterior prostate and (right panel) PBCre4/Foxa ${ }^{\text {loxp/loxp }}$ anterior prostate shows an increase in Foxc1 expression following Foxa1 knockout. WPMY-AR cells were used for co-immunoprecipitation experiments to determine whether Foxc1 physically interacts with AR. Western blotting results show that AR interacts with both endogenous (c) Foxc1 and exogenously expressed Foxc1 following transient transfection. 
estrogen receptor action in human breast. ${ }^{31}$ Recently, ChIP-Seq experiments on the glucocorticoid receptor showed that binding sites for this steroid receptor were enriched for forkhead binding sites, but the precise forkhead remains unidentified. ${ }^{32}$ Here we report that the conditional knockout of Foxal in the adult mouse prostate results in the loss of prostate-specific gene expression and the gain of at least 57 genes normally expressed in the seminal vesicle. Within this group of genes, we see expression of the androgen-regulated seminal vesicle secretion (SVS) genes. SVS2 is expressed in both the prostate and seminal vesicles, ${ }^{33,34}$ but SVSI and SVS3, SVS4, SVS5, and SVS6 are major androgen-regulated secretory proteins specific to the seminal vesicles. ${ }^{35}$ In keeping with this observation, we discovered that Foxa1 $\mathrm{KO}$ resulted in the increased expression of Nr4a3 and Foxc1 (see Supplementary Data), both of which are associated with mesodermal development. ${ }^{36,37}$ This switch of gene expression demonstrates that the prostate, which originates from endoderm, can be reprogrammed by the loss of Foxal to express androgen target genes that are specific to the seminal vesicle, which originates from mesoderm. This reprogramming is consistent with other studies that have demonstrated that not all AR-binding sites depend upon FOXA1 binding, and, in fact, loss of FOXA1 can reprogram AR to bind alternative promoters and result in the expression of AR-regulated genes that are normally repressed in the presence of FOXA1. ${ }^{38}$ In the mouse prostate, loss of FOXA1 appears to mediate the expression of seminal vesicle genes.

In order to determine possible alternative forkhead-binding partners for $\mathrm{AR}$, we looked both in our microarray analysis of the PB-Foxal mouse and the literature. Foxc1 was the most upregulated forkhead family member by microarray analysis, and analysis of Fox family members in prostate cancer cell lines had previously revealed that FOXA1 and FOXC1 were inversely expressed in androgen-dependent and independent cell lines. ${ }^{27}$ Although Foxal expression is largely restricted to endoderm, Foxal is detected in some mesodermally and ectodermally derived tissues. ${ }^{39,40}$ However, IHC staining of the seminal vesicle shows that the epithelial cells are Foxal negative but Foxc1 positive. Also, IHC staining of the prostate shows that the control prostates are Foxc1 negative but the conditional knockout of Foxal results in expression of Foxc1 in the prostate epithelium. Further, we demonstrate that the AR and Foxcl can directly interact suggesting that an AR/Foxcl complex is important in androgen action in the seminal vesicle. Unfortunately, no seminal vesicle cell lines have been developed that would allow us to confirm the AR/Foxcl complex regulates seminal vesicle genes that are enriched following Foxal knockout. However, it is interesting to note that $\sim 40 \%$ of primary prostate tumors are FOXA1 negative, and these negative prostate tumors exhibit relatively indolent behavior. ${ }^{41}$ It would be interesting to determine the extent to which FOXC1 and seminal vesicle-related genes are upregulated in this tumor cohort, as well as the ramifications of these potential gene expression changes, in regard to tumor biology.

Forkhead transcription factors are grouped into subfamilies (such as the Foxa subfamily) based on the extent of sequence conservation within the DNA-binding domain. Of the 43 forkhead proteins, Foxb1, Foxb2, and Foxc1 have the next most conserved DNA-binding motifs to Foxal. ${ }^{1}$ Taken together, this suggests a model where some of the organspecific target genes induced by the AR can, in part, be due to specific complexes between this receptor and a forkhead protein. Similarly, this model would apply to other steroid receptors that would partner with specific forkhead proteins to control steroid receptor action in multiple organs.

Supplementary Information accompanies the paper on the Laboratory Investigation website (http://www.laboratoryinvestigation.org)

\section{ACKNOWLEDGMENTS}

Grant support: 5R01-DK055748 to RJM; 5T32 DK007563-21 and 1T32 CA119925 to DJD; American Cancer Society Great Lakes Division-Michigan Cancer Research Fund Postdoctoral Fellowship to DJD; 1T32-CA119925 to MMG; 5R01-DK067049 to SWH; 5K01-DK085194 to NG.

\section{DISCLOSURE/CONFLICT OF INTEREST}

The authors declare no conflict of interest.

1. Hannenhalli S, Kaestner $\mathrm{KH}$. The evolution of Fox genes and their role in development and disease. Nat Rev Genet 2009;10:233-240.

2. Lai E, Prezioso VR, Smith E, et al. HNF-3A, a hepatocyte-enriched transcription factor of novel structure is regulated transcriptionally. Genes Dev 1990;4:1427-1436.

3. Clark KL, Halay ED, Lai E, et al. Co-crystal structure of the HNF-3/fork head DNA-recognition motif resembles histone H5. Nature 1993;364: 412-420.

4. Cirillo LA, Lin FR, Cuesta I, et al. Opening of compacted chromatin by early developmental transcription factors HNF3 (FoxA) and GATA-4. Mol Cell 2002;9:279-289.

5. Yan J, Xu L, Crawford G, et al. The forkhead transcription factor Foxl1 remains bound to condensed mitotic chromosomes and stably remodels chromatin structure. Mol Cell Biol 2006;26:155-168.

6. Zaret KS, Carroll JS. Pioneer transcription factors: establishing competence for gene expression. Genes Dev 2011;25:2227-2241.

7. Mirosevich J, Gao N, Matusik RJ. Expression of Foxa transcription factors in the developing and adult murine prostate. Prostate 2005;62:339-352.

8. Gao N, Ishii K, Mirosevich J, et al. Forkhead box A1 regulates prostate ductal morphogenesis and promotes epithelial cell maturation. Development 2005;132:3431-3443.

9. DeGraff DJ, $\mathrm{Yu} X$, Sun $\mathrm{Q}$, et al. The role of Foxa proteins in the regulation of androgen receptor activity. In: Tindall DJ, Mohler JL (eds). Androgen Action in Prostate Cancer, 2008.

10. Bernardo GM, Keri RA. FOXA1: a transcription factor with parallel functions in development and cancer. Biosci Rep 2012;32:113-130.

11. Gao N, Zhang J, Rao MA, et al. The role of hepatocyte nuclear factor-3 alpha (Forkhead Box A1) and androgen receptor in transcriptional regulation of prostatic genes. Mol Endocrinol 2003;17: 1484-1507.

12. Gupta A, Yu X, Case T, et al. Mash1 expression is induced in neuroendocrine prostate cancer upon the loss of Foxa2. Prostate 2013;73:582-589.

13. Yu X, Wang Y, DeGraff DJ, et al. Wnt/beta-Catenin activation promotes prostate tumor progression in a mouse model. Oncogene 2011;30: 1868-1879.

14. Grasso CS, Wu YM, Robinson DR, et al. The mutational landscape of lethal castration-resistant prostate cancer. Nature 2012;487:239-243. 
15. Barbieri $\mathrm{CE}$, Bangma $\mathrm{CH}$, Bjartell $\mathrm{A}$, et al. The mutational landscape of prostate cancer. Eur Urol 2013;64:567-576.

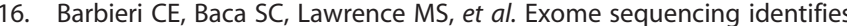
recurrent SPOP, FOXA1 and MED12 mutations in prostate cancer. Nat Genet 2012;44:685-689.

17. Mirosevich J, Gao N, Gupta A, et al. Expression and role of Foxa proteins in prostate cancer. Prostate 2006;66:1013-1028.

18. Gao N, LeLay J, Vatamaniuk MZ, et al. Dynamic regulation of Pdx1 enhancers by Foxa1 and Foxa2 is essential for pancreas development. Genes Dev 2008;22:3435-3448.

19. Wu X, Wu J, Huang J, et al. Generation of a prostate epithelial cellspecific Cre transgenic mouse model for tissue-specific gene ablation. Mech Dev 2001;101:61-69.

20. Muzumdar MD, Tasic B, Miyamichi K, et al. A global double-fluorescent Cre reporter mouse. Genesis 2007;45:593-605.

21. DeGraff DJ, Clark PE, Cates JM, et al. Loss of the urothelial differentiation marker FOXA1 is associated with high grade, late stage bladder cancer and increased tumor proliferation. PloS one 2012;7:e36669.

22. Yu X, Wang Y, DeGraff DJ, et al. Wnt/beta-catenin activation promotes prostate tumor progression in a mouse model. Oncogene 2011;30: 1868-1879.

23. Pfaffl MW. A new mathematical model for relative quantification in real-time RT-PCR. Nucleic Acids Res 2001;29:e45.

24. Tanner MJ, Welliver Jr. RC, Chen M, et al. Effects of androgen receptor and androgen on gene expression in prostate stromal fibroblasts and paracrine signaling to prostate cancer cells. PLoS One 2011;6:e16027.

25. Zhang J, Gao N, DeGraff DJ, et al. Characterization of cis elements of the probasin promoter necessary for prostate-specific gene expression. Prostate 2010;70:934-951.

26. Gerhardt J, Montani M, Wild $\mathrm{P}$, et al. FOXA1 promotes tumor progression in prostate cancer and represents a novel hallmark of castration-resistant prostate cancer. Am J Pathol 2012;180:848-861.

27. van der Heul-Nieuwenhuijsen L, Dits NF, Jenster G. Gene expression of forkhead transcription factors in the normal and diseased human prostate. BJU Int 2009;103:1574-1580.

28. Kurita T, Lee KJ, Cooke PS, et al. Paracrine regulation of epithelial progesterone receptor and lactoferrin by progesterone in the mouse uterus. Biol Reprod 2000;62:831-838.
29. Matusik RJ, Jin RJ, Sun $Q$, et al. Prostate epithelial cell fate. Differentiation 2008;76:682-698.

30. Gierer A. Molecular models and combinatorial principles in cell differentiation and morphogenesis. Cold Spring Harb Symp Quant Biol 1974;38:951-961.

31. Lam EW, Brosens JJ, Gomes AR, et al. Forkhead box proteins: tuning forks for transcriptional harmony. Nat Rev Cancer 2013;13:482-495.

32. Sahu B, Laakso M, Pihlajamaa $P$, et al. FoxA1 specifies unique androgen and glucocorticoid receptor binding events in prostate cancer cells. Cancer Res 2013;73:1570-1580.

33. Dodd JG, Kreis C, Sheppard PC, et al. Effect of androgens on mRNA for a secretory protein of rat dorsolateral prostate and seminal vesicles. Mol Cell Endocrinol 1986;47:191-200.

34. Harris $\mathrm{SE}$, Harris MA, Johnson CM, et al. Structural characterization of the rat seminal vesicle secretion II protein and gene. J Biol Chem 1990;265:9896-9903.

35. Ostrowski MC, Kistler MK, Kistler WS. Purification and cell-free synthesis of a major protein from rat seminal vesicle secretion. A potential marker for androgen action. J Biol Chem 1979;254: 383-390.

36. Wilm B, James RG, Schultheiss TM, et al. The forkhead genes, Foxc1 and Foxc2, regulate paraxial versus intermediate mesoderm cell fate. Dev Biol 2004;271:176-189.

37. DeYoung RA, Baker JC, Cado D, et al. The orphan steroid receptor Nur77 family member Nor-1 is essential for early mouse embryogenesis. J Biol Chem 2003;278:47104-47109.

38. Sahu $B$, Laakso $M$, Ovaska $K$, et al. Dual role of FoxA1 in androgen receptor binding to chromatin, androgen signalling and prostate cancer. Embo J 2011;30:3962-3976.

39. Sasaki H, Hogan BL. Differential expression of multiple fork head related genes during gastrulation and axial pattern formation in the mouse embryo. Development 1993;118:47-59.

40. Besnard V, Wert SE, Hull WM, et al. Immunohistochemical localization of Foxa1 and Foxa2 in mouse embryos and adult tissues. Gene Expr Patterns 2004;5:193-208.

41. Imamura $\mathrm{Y}$, Sakamoto $\mathrm{S}$, Endo $\mathrm{T}$, et al. FOXA1 promotes tumor progression in prostate cancer via the insulin-like growth factor binding protein 3 pathway. PLoS One 2012;7:e42456. 